\title{
Transperitoneal robotic-assisted laparoscopic radical prostatectomy and inguinal herniorrhaphy
}

\author{
David S. Finley $\cdot$ Dominic Savatta $\cdot$ Esequiel Rodriguez $\cdot$ \\ Adam Kopelan · Thomas E. Ahlering
}

Received: 20 June 2007 / Accepted: 5 December 2007 / Published online: 4 January 2008

(C) Springer London 2007

\begin{abstract}
Patients undergoing radical prostatectomy are at increased risk of development of post-operative inguinal hernias $(\mathrm{IH})$. We present the largest series of transperitoneal combined robotic-assisted laparoscopic prostatectomy (RALP) and IH. After IRB approval, data from patients undergoing RALP at two centers were prospectively entered into a database and analyzed. IH were repaired robotically via a transperitoneal route with mesh. Between June 2002 and May 2007, 837 RALPs were performed, 80 of which included combined IH repair $(9.6 \%)$, by two surgeons, T.A. and D.S. Forty-two patients $(52.5 \%)$ had IH on pre-operative exam. Twenty-four hernias were left, 32 right, and 24 bilateral. Twenty-two patients had prior ipsilateral or contralateral herniorrhaphy. After dissection of the hernia sac, a swatch of flat Marlex mesh $(n=22)$, a polypropylene mesh plug $(n=19)$, an Ultrapro hernia system $(n=7)$, a Proceed coated mesh $(n=19)$, a 3D-Max $(n=37)$, a combination of both umbrella and flat mesh $(n=3)$, or suture alone $(n=2)$ was used. Inguinal herniorrhaphy added approximately $15 \mathrm{~min}$ of operative time in all cases. There was one hernia recurrence over an average follow-up period of 12.5 months (0.2-52 months). There was one complication attributable to IH repair-a urine leak which was attributed to anastomotic stretch due to reperitonealization. Urological surgeons should perform a thorough inguinal exam during preoperative evaluation and intraoperatively to detect subclinical inguinal hernias. Inguinal
\end{abstract}

D. S. Finley $(\bowtie) \cdot$ E. Rodriguez $\cdot$ T. E. Ahlering

Department of Urology, University of California Irvine, 101 The City Dr, Bldg 55, Rm 304, Orange, CA 92868, USA

e-mail: finds@uci.edu

D. Savatta $\cdot$ A. Kopelan

Department of Surgery,

Newark Beth Israel Medical Center, Newark, NJ, USA herniorrhaphy at the time of RALP is safe and should be routinely performed.

Keywords Robotic-assisted laparoscopic radical prostatectomy $\cdot$ Laparoscopic prostatectomy $\cdot$ Inguinal hernia $\cdot$ Laparoscopic herniorrhaphy $\cdot$ Hernioplasty

\section{Introduction}

Approximately fifteen percent of patients undergoing radical prostatectomy (RRP) may develop a symptomatic inguinal hernia (IH) within the first post-operative year. Some patients have subclinical hernias that predate their surgery, of whom only a portion develops a de-novo IH as a post-operative complication. Both the actual post-operative incidence and the management of IH in RRP are controversial with some authors recommending routine repair of all IH during RRP. Walsh and others routinely repair IH at open radical retropubic prostatectomy [1-3]. To our knowledge, the current study represents the largest combined robotic prostatectomy and inguinal herniorrhaphy series reported.

\section{Materials and methods}

After written approval from our Institutional Review Boards, we prospectively collected data from 837 consecutive patients undergoing RALP.

Data points including preoperative history, physical characteristics, operative details, and post-operative length of hospital stay, complications, and recurrence rate were analyzed. All patients underwent preoperative and post-operative physical exam by one of the authors to identify $\mathrm{IH}$. 
Operative technique

After pre-operative confirmation of a negative urine culture, we make an attempt to examine the inguinal areas after establishing pneumoperitoneum. One surgeon, D.S., prefers to cover the mesh repair with peritoneum. If he finds a hernia defect with peritoneum exiting the abdominal cavity, he modifies the dissection by taking down the bladder with the attached peritoneum from the lower abdominal wall. The dissection involves extending the incision in the medial umbilical ligaments and urachus laterally as opposed to inferiorly.

After completion of transperitoneal RALP and pelvic lymph node dissection, both authors inspected the myopectineal orifice for the presence of a hernia. If IH was detected, it was repaired robotically by a modified Stoppa technique [4]. After dissection of the hernia sac, a flat piece of mesh, mesh plug, or mesh hernia system was secured over the defect with a combination of suture and staples. The mesh was trimmed to cover the myopectineal orifice. Two pieces of mesh were used for repair of bilateral hernias. The mesh was secured to Cooper's ligament inferiorly and along the superolateral borders of the rectus sheath. Mesh fixation lateral to the spermatic cord structures or posterior to iliopubic tract was avoided to prevent cutaneous nerve entrapment. Repairs done by T.A. were initially with Marlex mesh or plugs and are now exclusively with Proceed mesh. D.S. initially used Ultrapro mesh and now favors the 3-Dmax system and Proceed mesh, depending on the availability of peritoneum to cover the mesh. The 3-Dmax is form fitted for the hernia repair, but requires coverage with peritoneum. The 3-Dmax involves fixation in only three areas, two on the pubic bone and one on the medial edge of the mesh. If peritoneum is not available to close, usually due to prior hernia surgery or discovery of a hernia after the bladder has been taken down, we use the Proceed. A 5-mm laparoscopic ProTac device (Tyco USS, Norwalk, CT, USA) through the right lower quadrant assistant port was used to secure the flat mesh along the pubic bone earlier in the series of T.A. but was later abandoned in favor of suture alone; it is currently is still in use by D.S. No drains were used by T.A.; only two drains were left in the series by D.S. Reperitonealization was accomplished by individual preference: one author (T.A.) initially performed reperitonealization on several cases but later abandoned this practice after one anastomotic leak. A second author (D.S.) approximates the lateral peritoneal edges with suture or the Protac device. To avoid tension on the vesicourethral anastomosis, the midline peritoneum is not reapproximated. The peritoneum on the bladder side is approximated to the posterior rectus fascia inferior to the cut edge of the peritoneum. If the peritoneum is closed, it is important to close the peritoneum entirely to avoid internal hernias.

\section{Results}

Between June 2002 and May 2007, 837 consecutive patients underwent RALP at two hospitals by two surgeons, T.A. and D.S. Of these 837 patients, 80 underwent combined RALP and concurrent IH repair $(9.6 \%)$. The average age of the RAIH group was 62.2 years (range 43-80 years). Their average BMI was 26.6 (range 20.6-37.3). A total of one-hundred and six hernias were repaired (Table 1). Fiftysix IH were unilateral and 24 were bilateral. Twenty-four IH were left sided, 32 right sided, and 24 bilateral (Table 2). Fifteen patients with unilateral hernias had a previous ipsilateral $(n=7)$, contralateral $(n=4)$, or bilateral $(n=4)$ herniorrhaphy; four patients with bilateral hernias had a previous bilateral $(n=3)$ or unilateral $(n=1)$ repair (Table 1). Seventy-nine hernias were direct, nineteen

Table 1 Hernia cohort characteristics

\begin{tabular}{ll}
\hline Total RALP & 837 \\
No. patients & $80(9.6 \%)$ \\
Total hernias repaired & 106 \\
Age & 62.2 years \\
BMI & 26.6 \\
Prior repair & $19(23.8 \%)$ \\
\hline
\end{tabular}

Table 2 Hernia characteristics

\begin{tabular}{ll}
\hline Preoperative exam & \\
Hernia & 42 \\
Normal exam & 38 \\
Hernia side & \\
Left & $24(30 \%)$ \\
Right & $32(40 \%)$ \\
Bilateral & $24(30 \%)$ \\
Hernia type & \\
Direct & $79(76.7 \%)$ \\
Indirect & $19(18.4 \%)$ \\
Pantaloon & $4(3.9 \%)$ \\
Femoral & $1(1.0 \%)$ \\
Mesh type & \\
Plug & 19 \\
Marlex & 22 \\
Proceed & 19 \\
3D Max & 37 \\
Ultrapro & 7 \\
No mesh & 2 \\
Reperitonealization & $1 / 80(1.3 \%)$ \\
Avg length of stay & $12.5 \mathrm{mo}$ \\
Recurrence & \\
Avg Follow-up & $36 / 80$ \\
\hline
\end{tabular}


indirect, four pantaloon, and one femoral (Table 2). Three umbilical hernias were also detected and repaired with mesh. A swatch of flat Marlex mesh $(n=22 ;$ C.R. Bard, Cranston, NJ, USA), a polypropylene cone mesh plug ( $n=19$; C.R. Bard), an Ultrapro hernia system, $(n=7$; Johnson \& Johnson, Piscataway, NJ, USA), a Proceed coated mesh ( $n=19$; Ethicon, Piscataway, NJ, USA), a 3DMax $(n=37$; C.R. Bard), or a combination of both umbrella and flat mesh $(n=3)$ was secured with interrupted 3-0 Dexon or 3-0 Tevdek suture (Table 2). Two patients had small defects which were repaired with suture alone.

Inguinal herniorrhaphy added an average of $15 \mathrm{~min}$ of operative time to the RALP. No deaths occurred. There was one recurrent $\mathrm{IH}$ at an average follow-up period of 12.5 months (range: $0.2-52$ months). Another complication attributable to $\mathrm{IH}$ repair was one urine leak $(1.3 \%)$ which was thought to occur due to anastomotic stretch after reperitonealization (Table 2). There was no wound or mesh infection, and no significant thigh, groin, or scrotal pain/ parasthesia, or orchalgia was noted.

\section{Discussion}

In the first century $\mathrm{AD}$, Celsus was possibly the first physician author to describe the surgical treatment of IH [5]. It was not until 1949 that McDonald described IH concurrent with RRP [6]. In the 1960s, Nhyus significantly advanced herniology [7]. It was not until 1987 that Schlegel and Walsh reported the first large series of IH repair at the time of RRP using Nyhus's preperitoneal nonmesh technique [8]. We employed a variation of the preperitoneal mesh repair described by Stoppa.

Risk factors for IH are smoking, a high activity index, prior hernia, post-prostatectomy stricture, and lower abdominal incision [9-11]. A possible mechanism for the increased incidence of IH may result from disruption of the tensile strength of the transversalis fascia or alteration of the physiologic action of the so called "shutter mechanism" of the myopectineal orifice [7].

The incidence of IH found during laparoscopy is common. Watson et al. found a $13 \%$ incidence of asymptomatic $\mathrm{IH}$ in 100 consecutive patients undergoing laparoscopic procedures [12]. In a review of prostatectomy patients, Fukuta found a $20.4 \%$ incidence of radiographic IH on pre-operative CT scans [13]. Despite these findings, the significance of incidental IH is unclear. Whether or not incidental subclinical IH progresses to overt IH and over what time scale is unknown. Retrospective studies only identify clinically apparent hernias. In one of the few prospective studies in the literature, Nielsen and Walsh examined the myopectineal orifice of every patient undergoing RRP for evidence of a dilated internal inguinal ring or the presence of a direct or indirect hernia
[1]. They found 142 of 430 patients (33\%) had overt hernias or anatomic defects predisposing them to $\mathrm{IH}$. To date there has been no adequate prospective controlled study to define the natural history of asymptomatic IH.

Despite the foregoing problem most surgeons repair incidental IH concurrent with RP. Some surgeons delay a combined repair arguing that the risk of mesh infection is higher in the presence of a vesicourethral anastomosis. Our series using RALP and IH do not support this latter notion. In our current series we have not had any mesh infections. Others have found a similar low rate with laparoscopic $\mathrm{IH}$ [14-17].

In addition to infection, a reactive foreign body adhesion is a consideration with the use of mesh. We did not have any adhesions or small bowel obstructions in our series. One explanation for the formation of adhesion is direct contact of bowel with the mesh. Minimization of risk can be achieved by reperitonealization in which the mesh is excluded from contact with the bowel or by the use of a coated mesh (e.g. Proceed). Reperitonealization can be safely achieved without stretch on the vesicourethral anastomosis; the peritoneal edges are fixed laterally near the epigastrics while the midline is not closed, to avoid any anastomotic tension. Proceed is a multi-layered mesh which comprises monofilament polypropylene coated with polydiaxanone (PDS). A layer of oxidized regenerated cellulose fabric lines the visceral surface to minimize tissue adherence [18].

Robotic assistance offers several advantages over open IH repair. Because RALP employs $10 \times 3$ D magnification, the surgeon is able to inspect the internal inguinal anatomy in greater detail. In addition, the increased dexterity of the robotic instruments allow for more precise dissection of the hernia and straightforward suturing of the mesh. RAIH adds no more than 15 min to RALP.

\section{Conclusion}

Urological surgeons should be encouraged to perform a thorough inguinal exam during preoperative evaluation and intraoperatively to detect subclinical hernias. Inguinal herniorrhaphy done concurrently at the time of RALP is safe, with no added morbidity and should be routinely performed.

\section{References}

1. Nielsen ME, Walsh PC (2005) Systematic detection, repair of subclinical inguinal hernias at radical retropubic prostatectomy. Urology 66(5):1034-1037

2. Stranne J, Hugosson J, Lodding P (2006) Post-radical retropubic prostatectomy inguinal hernia: an analysis of risk factors with special reference to preoperative inguinal hernia morbidity and pelvic lymph node dissection J Urol 176:2072-2076 
3. Manoharan M, Vyas S, Araki M et al (2006) Concurrent radical retropubic prostatectomy and Lichtenstein inguinal hernia repair through a single modified Pfannenstiel incision: a 3-year experience. BJU Int 98:341-344

4. Stoppa RE, Rives JL, Warlaumont CR et al (1984) The use of Dacron in the repair of hernias of the groin. Surg Clin North Am 64:269-286

5. Papavramidou NS (2005) Christopoulou-Aletras H. Treatment of hernia in the writings of Celsus (first century AD). World J Surg 29(10):1343-1347

6. McDonald DF, Huggins C (1949) Simultaneous prostatectomy and inguinal herniorrhaphy. Surg Gynecol Obstet 89:621

7. Nyhus LM, Condon RE (1989) Inguinal hernia. In: Nyhus LM Condon's hernia, 3rd edn, Chap 3. Lippincott Williams \& Wilkins, Philadelphia, pp 74-77

8. Schlegel PN, Walsh PC (1987) Simultaneous preperitoneal hernia repair during radical pelvic surgery. J Urol 137(6):1180-1183

9. Lau H, Fang C, Yuen WK, Patil NG (2007) Risk factors for inguinal hernia in adult males: a case-control study. Surgery 141(2):262-266

10. Bielecki K, Puawaksi R (1988) Is cigarette smoking a causative factor in the development of inguinal hernia? Pol Tyg Lek 43:974

11. Twu C, Ou Y, Yang C, Cheng C et al (2005) Predicting risk factors for inguinal hernia after radical retropubic prostatectomy. Urology 66:814-818
12. Watson DS, Sharp KW, Vasquez JM et al (1994) Incidence of inguinal hernias diagnosed during laparoscopy. South Med J $87(1): 23-25$

13. Fukuta F, Hisasue S, Yanase M et al (2006) Preoperative computed tomography finding predicts for postoperative inguinal hernia: new perspective for radical prostatectomy-related inguinal hernia. Urology 68(2):267-271

14. Moon V, Chaudry GA, Choy C et al (2004) Mesh infection in the era of laparoscopy. J Laparoendosc Adv Surg Tech 14:349-352

15. Schmedt CG, Sauerland S, Bittner R (2005) Comparison of endoscopic procedures vs Lichtenstein and other open mesh techniques for inguinal hernia repair: a meta-analysis of randomized controlled trials. Surg endosc 19:188-199

16. Teber D, Erdogru T, Zukosky D et al (2005) Prosthetic mesh hernioplasty during laparoscopic radical prostatectomy. Urology 65:1173-1178

17. Stolzenburg JU, Rabenalt R, Dietel A et al (2003) Hernia repair during endoscopic (laparoscopic) radical prostatectomy. J Laparoendosc Adv Surg Tech 13(1):27-31

18. Novitsky YW, Harrell AG, Cristiano JA et al (2007) Comparative evaluation of adhesion formation, strength of ingrowth, and textile properties of prosthetic meshes after long-term intra-abdominal implantation in a rabbit. J Surg Res 140(1):6-11 